\title{
Structure Elucidation of a New Toxin from the Mushroom Cortinarius rubellus Using Gas Chromatography-Mass Spectrometry (GC-MS)
}

\author{
Ilia Brondz ${ }^{1,2}$ \\ ${ }^{1}$ Department of Biosciences, University of Oslo, Oslo, Norway \\ ${ }^{2} \mathrm{R} \&$ D Department of Jupiter Ltd., Ski, Norway \\ Email: ilia.brondz@bio.uio.no, ilia.brondz@gmail.com
}

Received October 28, 2013; revised November 24, 2013; accepted December 27, 2013

Copyright (C) 2013 Ilia Brondz. This is an open access article distributed under the Creative Commons Attribution License, which permits unrestricted use, distribution, and reproduction in any medium, provided the original work is properly cited.

\begin{abstract}
Cortinarius orellanus (Fries) and C. rubellus (Cooke), which were formerly also known as C. speciosissimus, are poisonous mushrooms containing the toxin orellanine and several degradation products of orellanine, including orelline and orellinine. Mass intoxication by poisonous mushrooms was observed in Poland in 1952-1957 [1]. In 1957, the cause of these outbreaks was described by Grzymala as poisoning by a member of the Cortinarius family. The toxin orellanine was first isolated from C. orellanus by Grzymala in 1962; the chemical structure of orellanine was later determined to be $3,3^{\prime}, 4,4^{\prime}$-tetrahydroxy-2,2'-bipyridine- $N, N^{\prime}$-dioxide. Poisoning with $C$. orellanus and $C$. rubellus has a very specific character. The first symptoms of intoxication usually do not appear until 2 - 3 days after ingestion, but in some cases intoxication appears after three weeks. The target organ for the toxin is the kidney. Histologically, it is easy to record the specific damage. The presence of degradation products of orellanine in kidney can be confirmed chromatographically, suggesting that the cause of poisoning is orellanine. However, the presence of orellanine in the blood of intoxicated persons has not been directly detected. A specific model was developed by Brondz et al. for the detection of orellanine, orelline, and orellinine in animal stomach fluids [2-4]. The hypothesis that the fungal toxin orellanine as a diglucoside can be transported from the digestive system by the blood to the kidney could not be supported. The toxin orellanine as a diglucoside is very unstable in an aqueous acidic environment. However, in the present study, it was possible to record an additional substance in animal stomach fluids using GC-MS after ingestion of $C$. rubellus. This substance, which has been named rubelline, is part of a toxic mixture in C. orellanus and C. rubellus and is closely related to orellanine. The structure of rubelline is more suitable than orellanine for absorption from the digestive tract and for transport in the blood. The presented hypothesis is that rubelline is absorbed in the digestive tract and transported in the blood to the kidney, where it is biotransformed to orellanine and accumulated to toxic levels. The process of biotransformation is in itself also damaging for the kidney and liver. GC-MS instrumentation enables the separation of substances in biological samples and in the extract from C. rubellus. The GC-MS with SMB technique was used to record the mass ion and to record a detailed fragmentation picture.
\end{abstract}

Keywords: Gas Chromatography; Mass Spectrometry

\section{Introduction}

Humankind has known poisoning by toxic mushrooms from the early stages of civilization. The attraction of mushrooms as delicious nutritious food has been known to humanoids starting from humanoid apes. Nonbotanical grouping simply classifies mushrooms as edible, nonedible, and toxic [5]. In the Roman Empire, toxic mushrooms became a political tool and were used for the as- sassination of highly ranked politicians including some imperators. Mithradates VI (Greek: Mi $\theta \rho \alpha \delta \alpha ́ \tau \eta \varsigma)$, King of Pontus was known for his extensive use of toxic mushrooms for poisoning and as an antidote [5]. In the Roman Empire, the slave-cooks were obliged to eat meals containing mushrooms one day ahead of an imperator-if the slave-cook was well one day after, the meal was served to the imperator. However, even this precaution could not save the life of the imperator if $C$. orella- 
nus or C. rubellus was laid on the table as a part of the meal, because the latent period between ingestion of these mushrooms and the appearance of the first symptoms of poisoning can stretch up to two or three weeks [2-6].

Intoxication by $C$. orellanus or $C$. rubellus is characterized by a loss of kidney function, the so-called "orellanus syndrome" with an extremely long-latent period of up to three weeks [2-6] before renal failure, and often extending to severe renal damage and the need for dialysis, or even to death as a result. Presently, the significance of ingestion of small doses of these toxic mushrooms is not clear, especially if the quantity of ingested toxin does not exceed toxic levels, but is repeated within short intervals (in one or two days), or within long intervals (in weeks or months). Will the kidney recover from intoxication or will there be a long-term impairment, for example for months or years? Will the damage be repaired in the long term or will repetitive ingestion of small doses, even over long intervals, lead to cumulative effects and result in kidney function failure or even death? These are not only academic questions, but also more practical ones because debris of C. orellanus or C. rubellus can appear in the mixtures of "forest mushrooms" prepared and sold commercially. The appearance of an increased number of patients with kidney failure can be explained by these intoxications or by intoxications with pesticides such as paraquat and diquat, which are similar in chemical formula and in their picture of intoxication.

Different toxins are blamed for the intoxication by $C$. orellanus or C. rubellus; for example, the cortinarines [7]. However, structures of cortinarines as described by Tebbett and Caddy [7] have been criticized by Matthies and Laatsch [8]. The existence of so-called cortinarines is highly questionable; thus, intoxication by cortinarines is also questionable. However, one hypothesis that persists is that the responsible poisonous substance is orellanine and partly its degradation products (Figure 1).

However, to our knowledge, there is no published literature that describes the presence of orellanine in blood samples after cases of intoxication by C. orellanus or $C$. rubellus. The description of the presence of orellanine in kidney after a case of intoxication by $C$. orellanus or $C$. rubellus is given by using thin layer chromatography (TLC) [9], in which Rohrmoser et al. stated: "Orellanine can be detected after a relatively long period following poisoning by performing a simple thin layer chromatography technique using small quantities of renal biopsy material. No toxin was found in urine or blood samples." In another report, the presence of orellanine was established indirectly: "By assaying orellanine in the plasma and two renal biopsies of patient after specific photodecomposition into a non-toxic metabolite called orelline" [10]. This indirect determination of orellanine by using an evidence of the presence of its degradation product orelline has several weaknesses.

1) Quantitative determination was not supported by dynamic and quantitative determination of output from the performed reaction.

2) There is no evidence that a substance other than orellanine was not a source for the resulting product "orelline" in these experiments.

3) There is no evidence that the orelline was absent in the blood before the so-called "degradation reaction by irradiation". In other words, the orelline could have come directly from the mushrooms themselves. The orelline may be present in the mushroom basidiocarps as a secondary metabolite and as a degradation product of orellanine. This issue is the greatest weakness in the study.

4) The argument that the orelline resulted entirely from the photodegradation reaction is not supported in [10] and cannot be supported by this way.

Antkowiak et al. described the chemical structure of orellanine as 3,3',4,4'-tetrahydroxy-2,2'-bipyridine- $N, N^{\prime}$ dioxide [11]; later, Holmdahl et al. supported the authenticity of this chemical structure by ${ }^{1} \mathrm{H}-\mathrm{NMR}$ and ${ }^{13} \mathrm{C}$ NMR spectra analyses [12].

The presence of orellanine in the mushrooms as a diglucoside was described in [13]; however, the NMR spectrum described here was not in good agreement with the theoretically calculated spectrum described in [4].

In [14], Herrman et al. supported the existence of orellanine Figure 2 as diglucopyranoside and suggested that orellanine-4,4'-diglucopyranoside is unstable in an aqueous acidic environment.

This can explain the inability to detect orellanine-4, 4'-diglucopyranoside in stomach fluids in experiments described in [2-4]. In the present study, a more careful approach to analyses of stomach fluids was taken by optimizing gas chromatography-mass spectrometry (GCMS) with supersonic molecular beams (SMB). Basidiocarps from C. rubellus were examined for the presence of a new toxin/s. The priority was to detect the substance/s that could be present in stomach fluid as a result of digestion of C. rubellus and that could be absorbed and transported in the blood to the kidney. It has been shown [14-16] that semisynthetic thermolabile heterocyclic substances can be successfully analyzed by using GC-MS with SMB techniques. By comparison with conventional GC-MS, the temperature of the analyses in GC-MS with SMB is significantly lower, the time of a single analysis is shorter, the gas flow in the capillary column is higher, and a contact-free fly-through EI ion source technique prevents contamination. The GC-MS with SMB technique was used to record the mass ion and to record a detailed fragmentation picture. All these advantages were used for tentative elucidation of the structure of rubelline. 
<smiles>O=c1ccn(O)c(-c2c(O)c(=O)ccn2O)c1O</smiles>

Orellanine

$$
\begin{gathered}
\text { Chemical Formula: } \mathrm{C}_{10} \mathrm{H}_{8} \mathrm{~N}_{2} \mathrm{O}_{6} \\
\text { Exact Mass: 252,04 } \\
\text { Molecular Weight: } 252,18
\end{gathered}
$$<smiles></smiles>

Orellinine

Chemical Formula: $\mathrm{C}_{10} \mathrm{H}_{8} \mathrm{~N}_{2} \mathrm{O}_{5}$ Exact Mass: 236,04 Molecular Weight: 236,18<smiles>O=c1cc[nH]c(-c2[nH]ccc(=O)c2O)c1O</smiles>

Orelline

Chemical Formula: $\mathrm{C}_{10} \mathrm{H}_{8} \mathrm{~N}_{2} \mathrm{O}_{4}$ Exact Mass: 220,05 Molecular Weight: 220,18

2<smiles>C[14CH2][14CH2]Oc1cc[n+]([O-])c(-c2c(O)c(O)cc[n+]2[O-])c1O</smiles>

4

Figure 1. The poisonous substances in Cortinarius orellanus and C. rubellus are orellanine structures 1 and 4 , and the degradation products orellinine structure 2 and orelline structure 3.

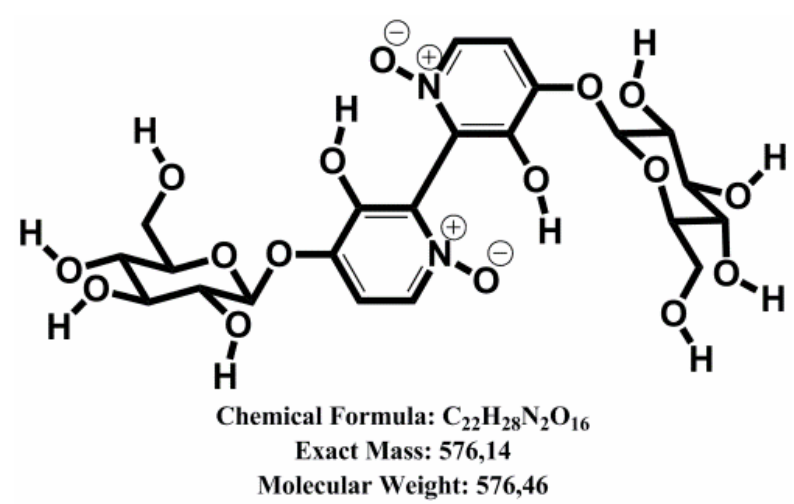

Figure 2. The structure of orellanine diglucopyranoside as presented in [13].

\section{Materials and Methods}

\subsection{Biological Material, Standards, and Extracts for Biological Testing}

Mushrooms C. rubellus were kindly provided by Jupiter Ltd., Norway and freeze-dried to a constant weight as described in [4]. The procedure for extraction and isolation of their toxins was conducted as described in [17]. The orellanine standards were donated by Jupiter Ltd., Norway. Cells of Chlamydomonas reinhardtii were prepared as previously described in detail $[18,19]$.

\subsection{Biological Testing of Toxicity}

The test model used in the experiment was as previously described $[18,19]$.

\subsection{Instrumentation and Conditions}

GC-MS instrumentation enabling the separation of substances in biological samples and in the extract from $C$. rubellus is described in detail in [16]. The extracted substances were dissolved in methanol p.a. quality (Merck, Darmstadt, Germany). The capillary column used was an Rxi-5Sil MS low polarity phase; Crossbond 1,4-bis (dimethylsiloxy) phenylene dimethyl polysiloxane catalog no. $13620,15 \mathrm{~m}$, ID $0.25 \mathrm{~mm}$, film thickness $0.25 \mu \mathrm{m}$ (Restek, Bellefonte, PA, USA). The column was reduced to about $4.5 \mathrm{~m}$ in laboratory. The helium column flow rate was $8 \mathrm{~mL} / \mathrm{min}$. Samples $(1 \mu \mathrm{L})$ at an approximate concentration of $20 \mathrm{ppm}$ of dry mass were injected with a split ratio of 10:1 using a Varian 1079 injector operated at $150^{\circ} \mathrm{C}$. The $\mathrm{GC}$ oven was programmed from $130^{\circ} \mathrm{C}$ to $330^{\circ} \mathrm{C}$ at $20^{\circ} \mathrm{C} / \mathrm{min}$.

Ion source degradation was prevented by virtue of a contact-free fly-through EI ion source [16], and degradation of substances in the sample was further avoided because of the use of a short column and a high gas flow rate.

The MS instrument was operated in an electron impact (EI) mode at $70 \mathrm{eV}$. The mass spectra between $\mathrm{m} / \mathrm{z} 50$ and $\mathrm{m} / \mathrm{z} 700$ were obtained at a scan speed of $200 \mathrm{~m} / \mathrm{z}$ unit scan/s with a mass resolution corresponding to 1 unit at half peak height. Separation and detection of substances in the stomach of Mus rattus L. are shown in the 
reconstructed ion chromatogram (RIC) in Figure 3 and the substances isolated directly from $C$. rubellus in Figure 4. The mass spectra of substances shown in Figure 3 are shown in Figures 5-8.

\section{Results and Discussion}

\subsection{Biological Testing of Toxicity}

The toxicological test is described in $[18,19]$. The thres- hold concentration of dry substance rubelline from $C$. rubellus used for inhibition of growth of Chlamydomonas reinhardtii cells was $10 \mathrm{ppm}$. Only qualitative results were recorded. Cell movement was abolished after exposure to the extract. This abolition of cell movement was the result of flagella blockage [20]; the authors of [20] noted: "The evidence is strongest for a role in triggering the shedding of flagella by the unicellular alga Chlamydomonas reinhardtii under acid stress. Rapid kinetic

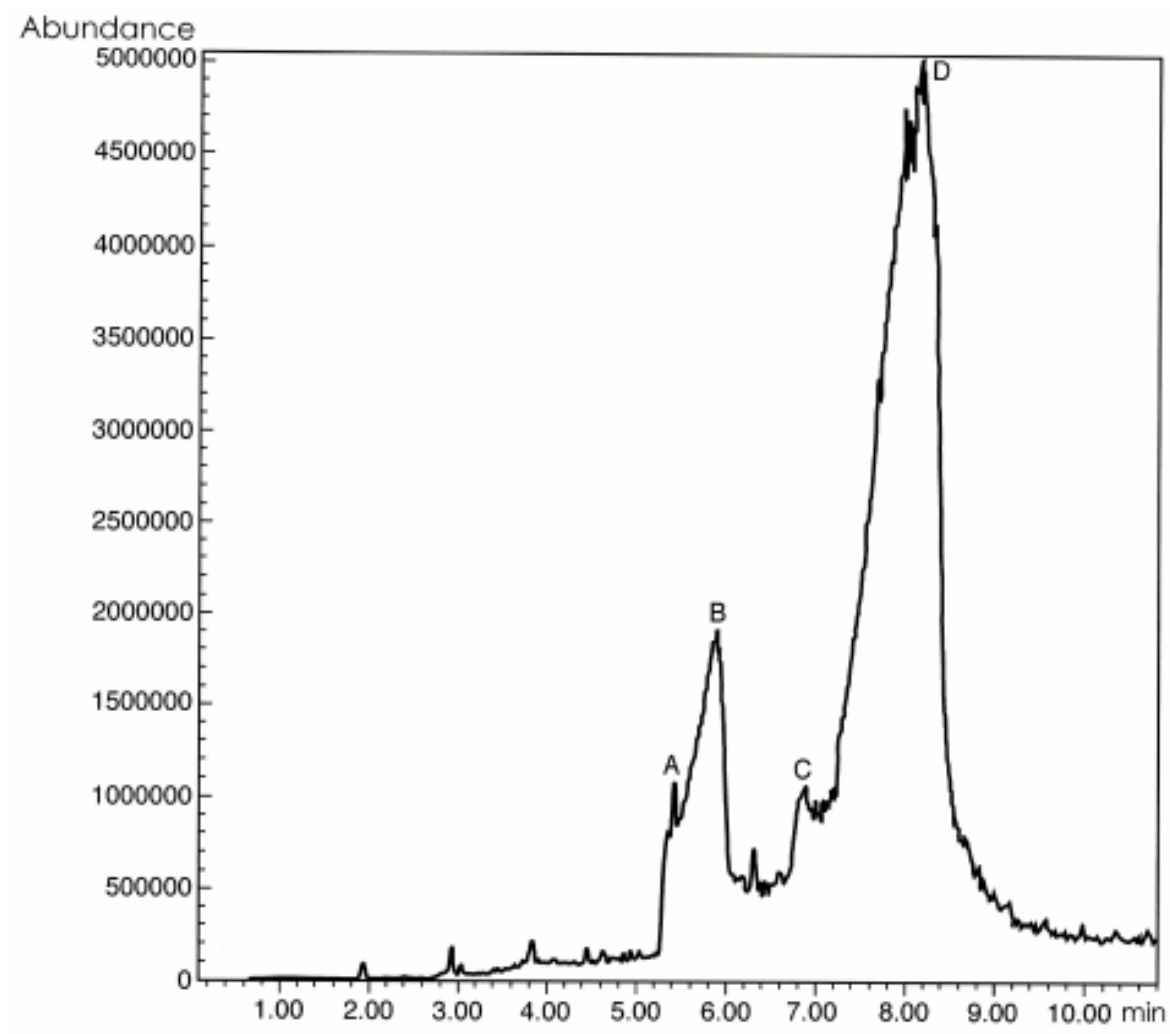

Figure 3. An RIC of the analyzed stomach fluid is shown: peak A is rubelline, peak B is orelline, peak C is orellinine, and peak $D$ is orellanine.

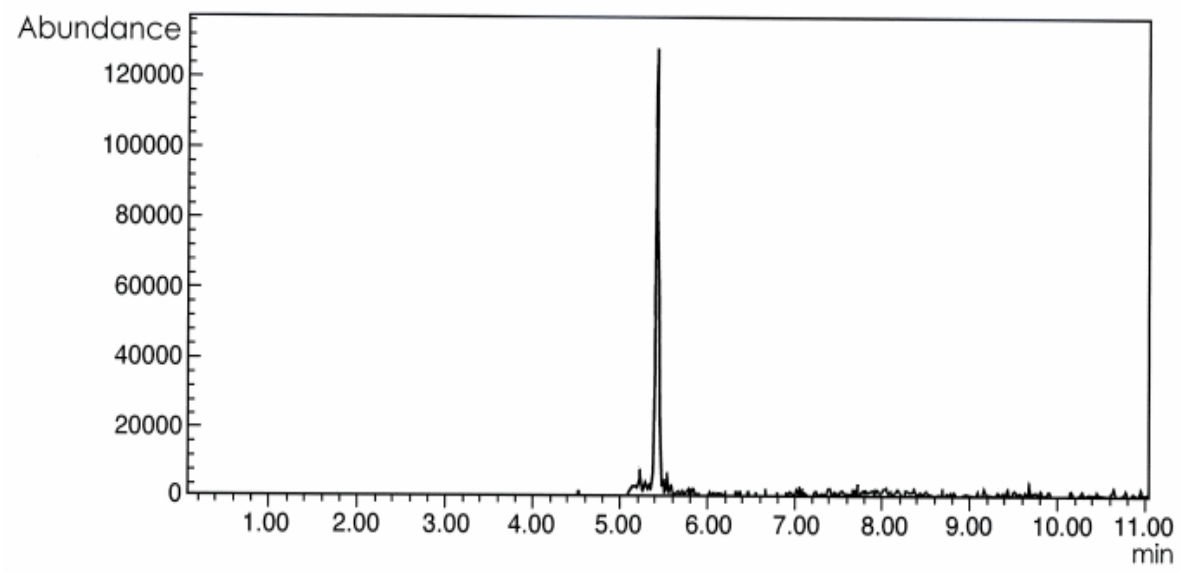

Figure 4. An RIC of the rubelline. 


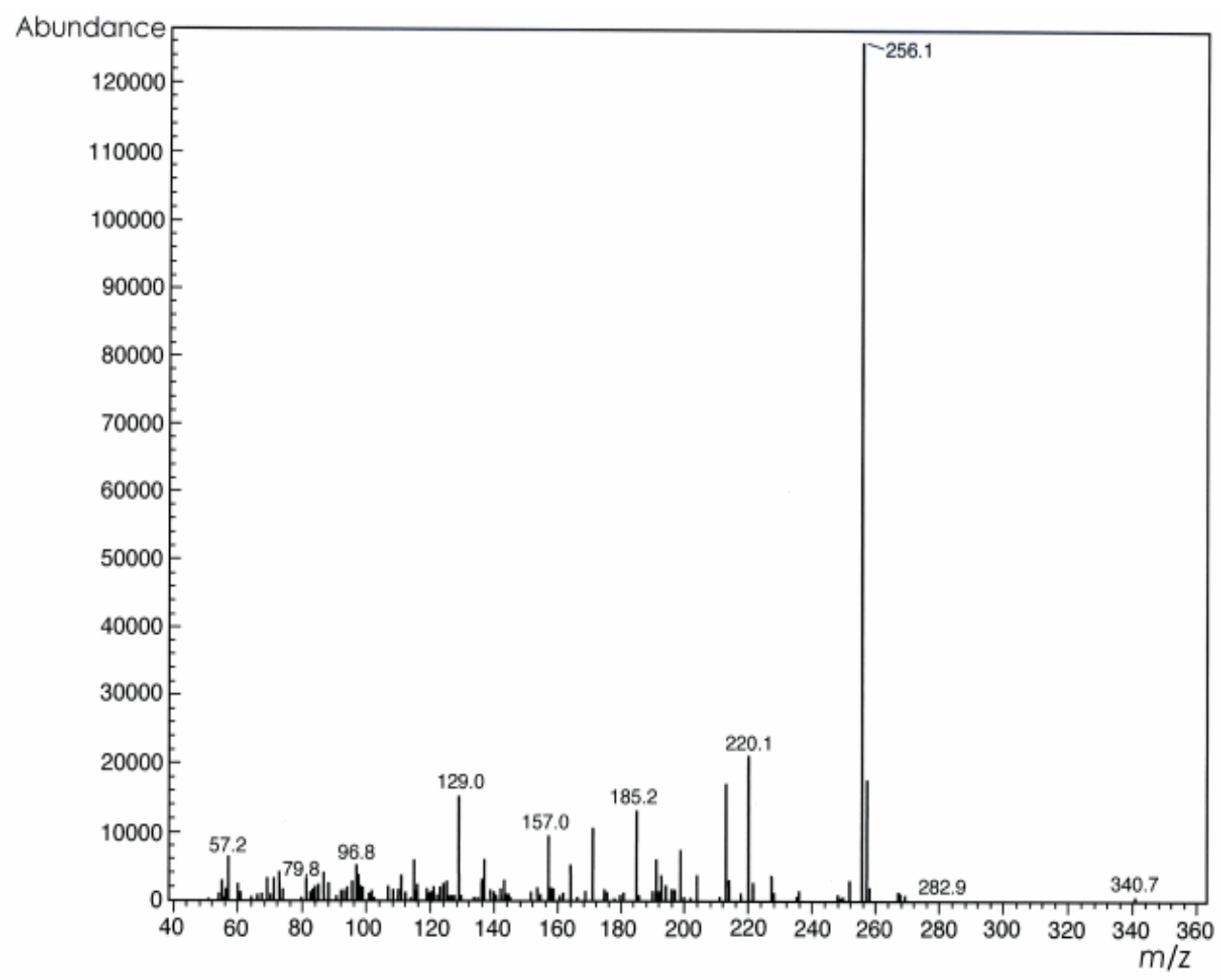

Figure 5. Mass spectrum of rubelline.

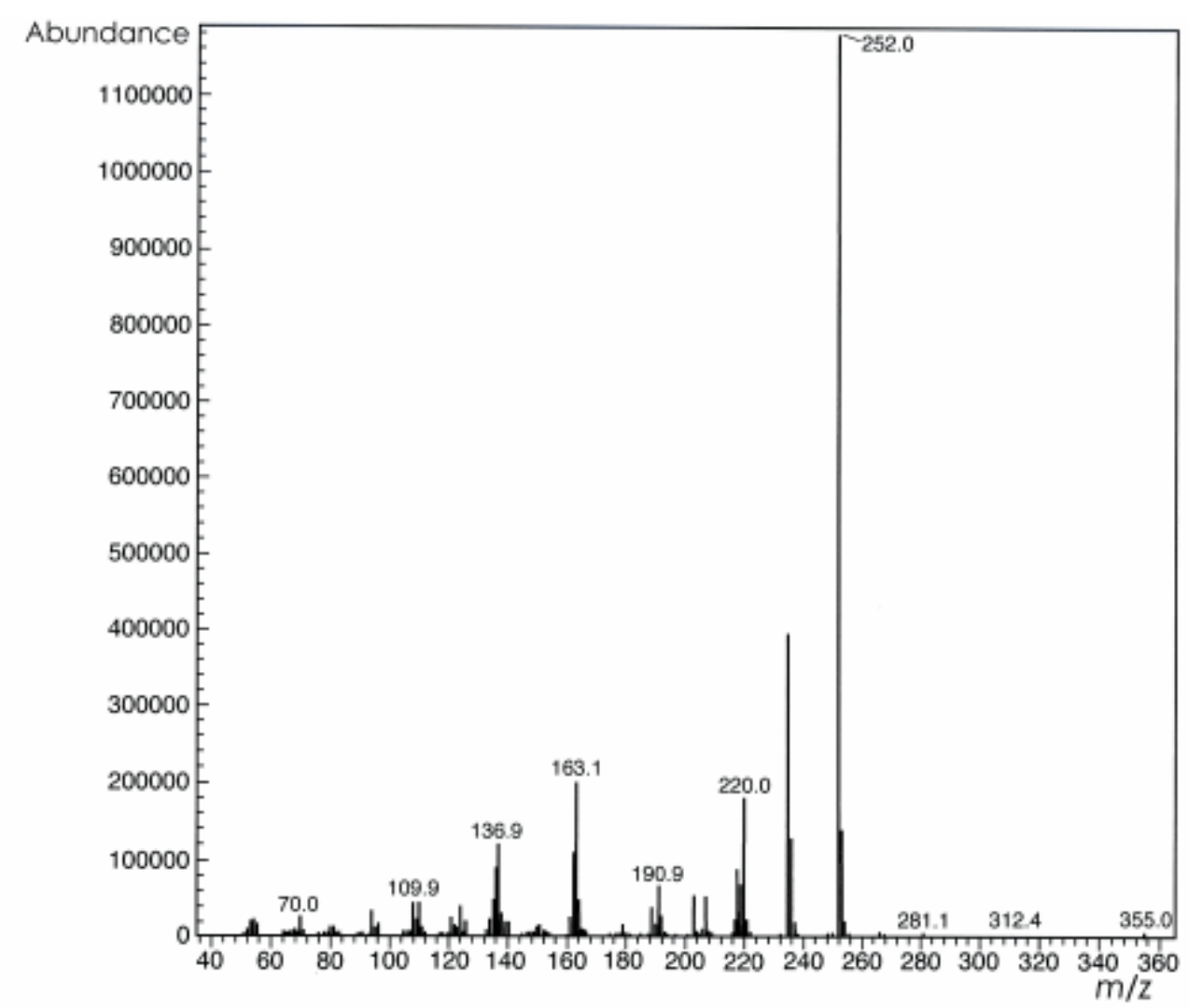

Figure 6. Mass spectrum of orellanine. 


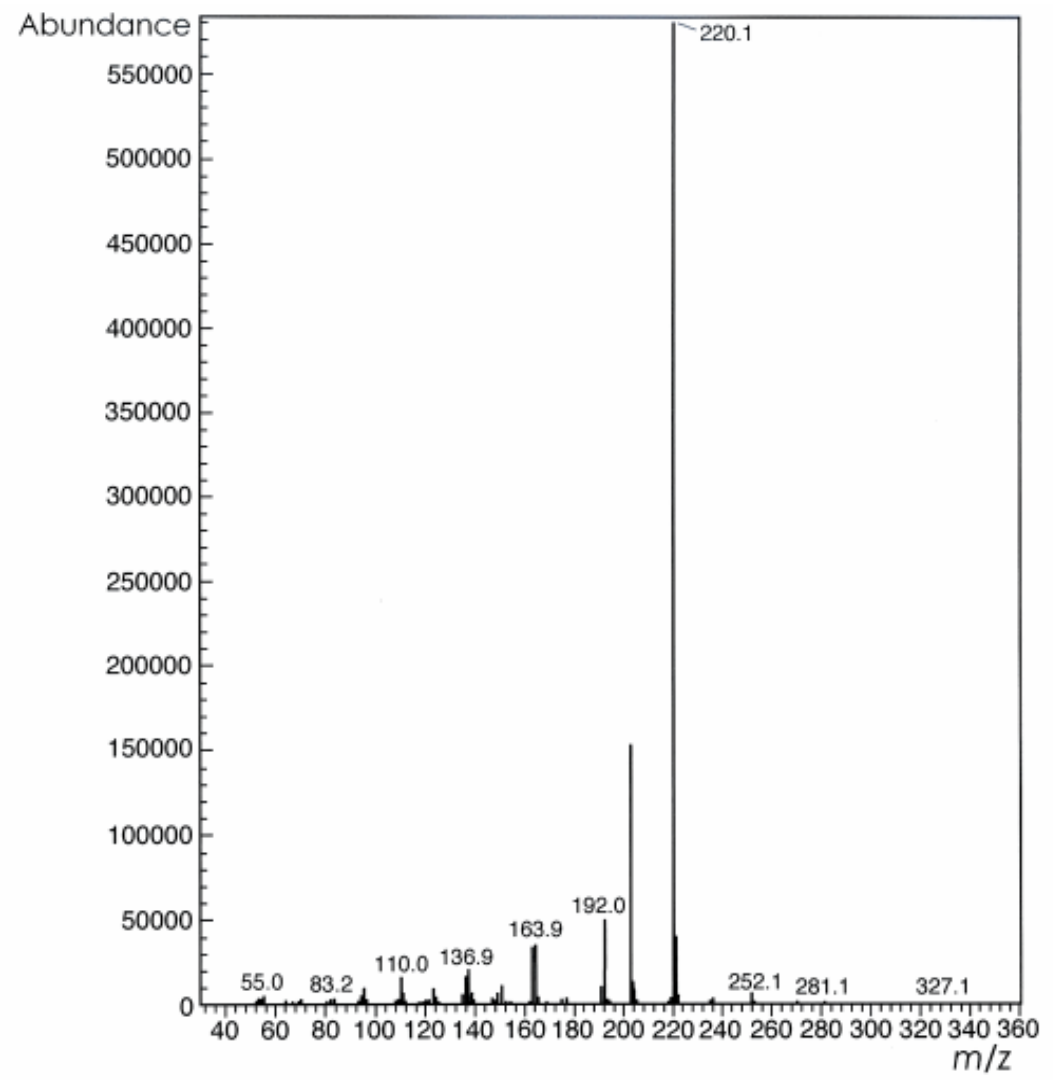

Figure 7. Mass spectrum of orelline.

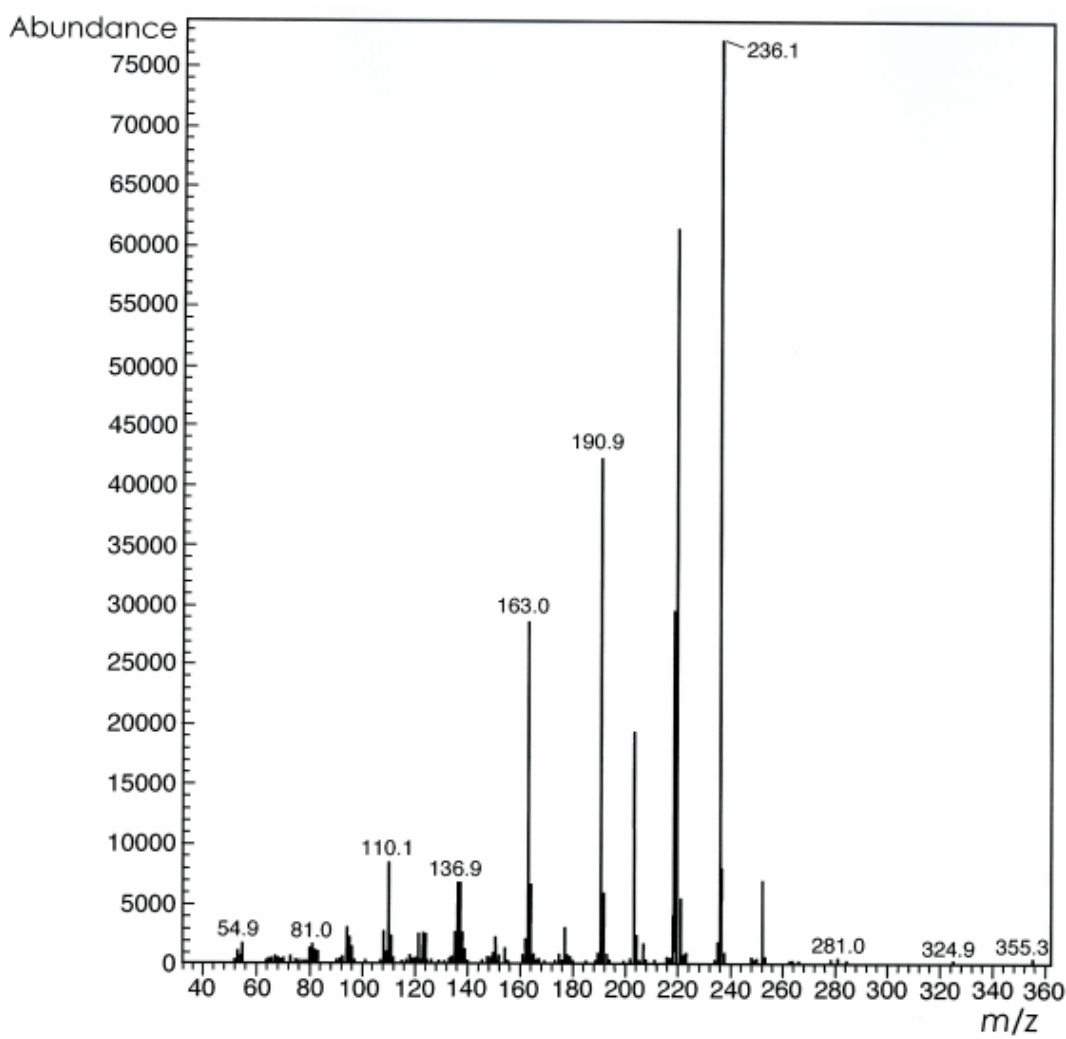

Figure 8. Mass spectrum of orellinine. 
analysis indicates that phosphoinositide hydrolysis occurs within half a second and could trigger the rapid loss of flagella." Most of the cells lost their chlorophyll and the cell lysis began as a result of exposure to the toxin.

Figure 1 presents orellanine and its degradation products. Degradation of orellanine occurs in the bodies of intoxicated persons and other organisms after ingestion of the mushrooms, in the basidiocarps of the mushrooms themselves, and during extraction.

The diglucoside of orellanine is an unstable substance (Figure 2) and degrades to the monoglucoside and orellanine itself $[13,14]$. The diglucoside of orellanine is particularly unstable in aqueous solutions at acidic $\mathrm{pH}$ [14] and this is probably the reason that it was not detected in experiments described in [2-4]. However, if the molecular structure exists, there is little chance that it will be absorbed and transferred into the blood stream because of its extreme instability in the aqueous acidic conditions [14] in the stomach.

\subsection{Analysis of Stomach Fluid from Mus rattus $\mathbf{L}$.}

The time of digestion of a meal containing C. rubellus in the stomach of the mouse Mus rattus L. was reduced to 1 $\mathrm{h}$. The injector temperature in $\mathrm{GC}$ was set at $150^{\circ} \mathrm{C}$ (low by comparison with previous analyses) to minimize the degradation at the injector. The VF-5HT column, I.D. $0.25 \mathrm{~mm}$, film thickness $0.1 \mu \mathrm{m}$, and $4 \mathrm{~m}$ length (Varian, Middelburg, The Netherlands) was changed to an Rxi5Sil MS low polarity phase; Crossbond 1,4-bis (dimethylsiloxy) phenylene dimethyl polysiloxane, catalog no. $13620.15 \mathrm{~m}$, I.D. $0.25 \mathrm{~mm}$, film thickness $0.25 \mu \mathrm{m}$ (Restek) column, which was reduced in length to about $4.5 \mathrm{~m}$ in the laboratory. A high flow rate in the capillary was used to reduce the time of analysis and avoid degradation of the analytes.

An RIC of stomach fluid from Mus rattus L. is shown in Figure 3.

An RIC of authentic rubelline donated by Jupiter Ltd. is shown in Figure 4.

The retention time for the peak A in the chromatogram in Figure 3 is the same as the retention time of standard in Figure 4. The mass spectrum shown in Figure 5 is the mass spectrum of peak A shown in Figure 3 and it is similar to the mass spectrum derived from the standard shown in Figure 4.

The $m / z$ of rubelline is 4 units greater than the $m / z$ of orellanine eluted under peak $D$; the mass spectrum of the substance eluted under peak D is shown in Figure 6.

The mass spectrum shown in Figure 6 is characteristic of orellanine.

The substance eluted under peak B yielded the mass spectrum shown in Figure 7.

The substance eluted under peak $\mathrm{C}$ has the mass spec- trum shown in Figure 8.

It appears as if the sequence of elution progresses from less polar and lower boiling substances to more polar and higher boiling substances as shown in Table 1. This was expected using the Rxi-5Sil column for GC-MS analyses. The toxicity from orelline to orellanine progressed from the low-molecular-weight orelline as nontoxic to the higher-molecular-weight orellanine as the most toxic in the sequence. Because of this, it is expected that rubelline, with the highest molecular weight, is most toxic, as was described for trivalent heterocyclic nitrogen and pentavalent nitrogen alkaloids [21,22]. The hypothesis is: the toxicity from orelline to rubelline should progress from the low-molecular-weight orelline as nontoxic to the higher-molecular-weight rubelline as the most toxic in the sequence.

Examples of biological transformation of less polar alkaloids to polar-charged molecules are known. These polar-charged molecules have a higher boiling point. An example of this is the transformation of (S)-canadine to berberine Figure 9.

The tentatively elucidated structural formula for rubelline is shown in Figure 10.

Orellanine and rubelline are similar and they are related structurally to paraquat and diquat. Paraquat and diquat are nonselective bipyridyl herbicides; rubelline exhibits the herbicide cytotoxic effect. As stated by Jones and Vale [23] "Diquat is a potent redox cycler and is readily converted to a free radical which, in reaction with molecular oxygen, generates superoxide anions and subsequently other redox products. These products can induce lipid peroxidation in cell membranes, and potentially cause cell death." The cytotoxic effect of rubelline was tested in a specific model [19] on unicellular algae Chlamydomonas reinhardtii. The model was developed to test the ability of primaquine and quinocide to interfere with the electron transport chain, to scavenge electrons, and to produce peroxides and radicals in the living cell.

\section{Conclusions}

1) The molecular weight of rubelline is 256 and the structure of rubelline was tentatively elucidated using GC-MS,

2) Rubelline is less polar than orellanine and is consequently more suitable for absorption in the stomach and digestive tract and transport in the blood to reach target organs such as the liver and the kidneys.

3) Rubelline is converted in mitochondria to orellanine and gradually concentrates in the kidney.

4) The biotransformation of rubelline to orellanine impairs kidney function by interference with electron transport, by production of radicals and peroxides, and 
Table 1. The characteristics for: rubelline, orelline, orellinine and orellanine.

\begin{tabular}{|c|c|c|c|c|}
\hline Peak & $\mathbf{A}$ & B & $\mathbf{C}$ & $\mathbf{D}$ \\
\hline & Rubelline: CAS No. & Orelline: CAS No: $72016-31-0$ & Orellinine: CAS No.: 98726-96-6 & Orellanine: CAS No.: 37338-80-0 \\
\hline & Molecular Weight: 256.1 & Molecular Weight: 220.1815 & Molecular Weight:236.1809 & Molecular Weight:252.17 \\
\hline \multicolumn{4}{|c|}{ Boiling Point: not known Boiling Point: $257.4^{\circ} \mathrm{C}$ at $760 \mathrm{~mm} \mathrm{Hg}$ B } & Boiling Point: $834.615^{\circ} \mathrm{C}$ at $760 \mathrm{~mm} \mathrm{Hg}$ \\
\hline & Formula: $\mathrm{C}_{10} \mathrm{H}_{12} \mathrm{~N}_{2} \mathrm{O}_{6}$ & Formula: $\mathrm{C}_{10} \mathrm{H}_{8} \mathrm{~N}_{2} \mathrm{O}_{4}$ & Formula: $\mathrm{C}_{10} \mathrm{H}_{8} \mathrm{~N}_{2} \mathrm{O}_{5}$ & Formula: $\mathrm{C}_{10} \mathrm{H}_{8} \mathrm{~N}_{2} \mathrm{O}_{6}$ \\
\hline \multicolumn{5}{|c|}{ Retention time } \\
\hline & Rubelline & Orelline Orellinine & \multicolumn{2}{|l|}{ Orellanine } \\
\hline & $5.38 \mathrm{~min}$ & $5.84 \mathrm{~min}$ & $6.92 \mathrm{~min}$ & $8.05 \mathrm{~min}$ \\
\hline
\end{tabular}

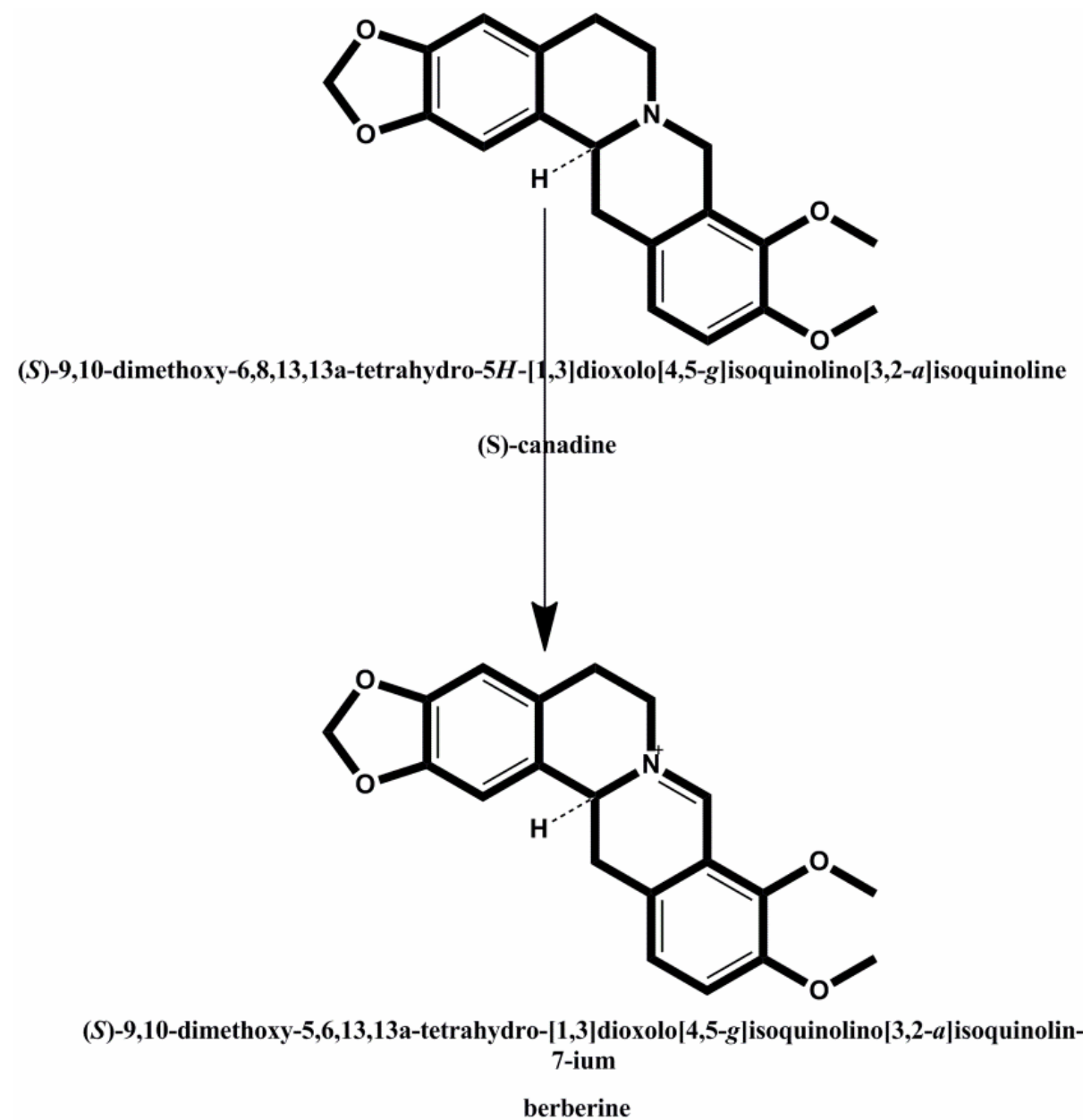

Figure 9. The transformation of ( $S$ )-canadine to berberine.

by direct toxicity.

5) Orellanine is a toxic substance that gradually concentrates in the target organs until the concentration becomes such that critical irreversible damage occurs.

6) The process of biotransformation from rubelline to orellanine takes time and this may explain the long-latent period of Cortinarius poisoning. Individual differences in enzyme activity in different patients may explain the difference in the latent period: in some patients this is 2 days, in others up to 3 weeks.

7) The discovery of a molecule with $\mathrm{m} / \mathrm{z} 256$ suggests that a molecule with $\mathrm{m} / \mathrm{z} 580$ may exist. A hypothetical molecule with $\mathrm{m} / \mathrm{z} 580$ should have the structure shown in Figure 11.

\section{Acknowledgements}

The author is grateful to Jupiter Ltd., Norway, for financial support, biologic material and standards, to Professor Aviv Amirav, School of Chemistry, Tel Aviv University, Tel Aviv, Israel for technical assistance with gas chromatography-mass spectrometry and to Anton Brondz, 


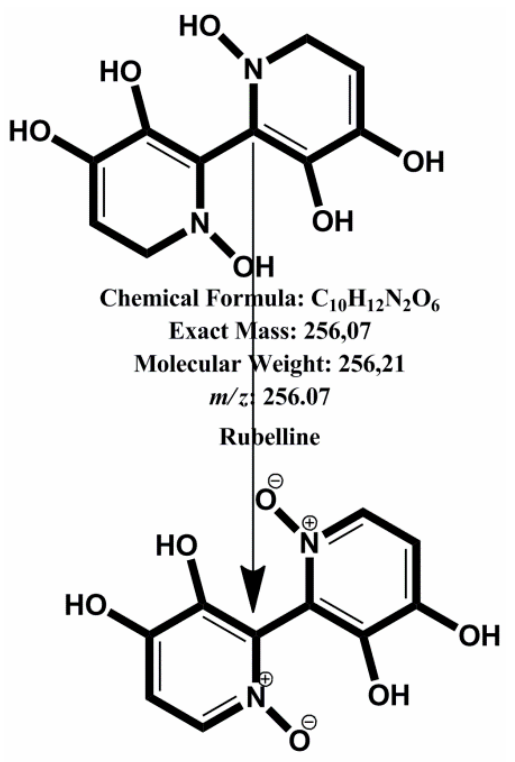

$$
\begin{gathered}
\text { Chemical Formula: } \mathrm{C}_{10} \mathrm{H}_{8} \mathrm{~N}_{2} \mathrm{O}_{6} \\
\text { Exact Mass: 252,04 } \\
\text { Molecular Weight: 252,18 } \\
m / z: 252.04 \\
\text { Orellanine }
\end{gathered}
$$

Figure 10. The possible biotransformation of rubelline to orellanine.

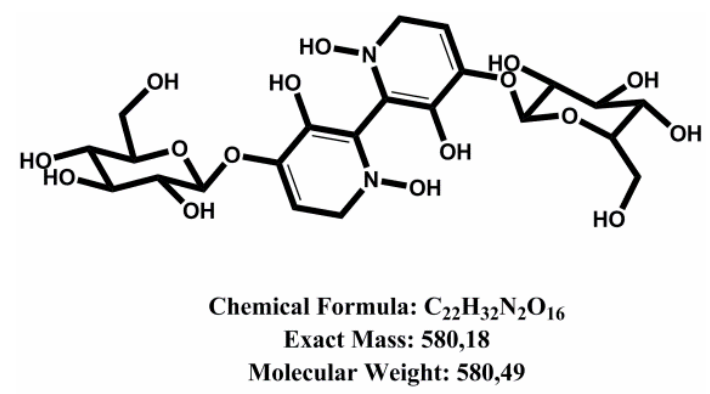

Figure 11. The hypothetical molecule with $\mathrm{m} / \mathrm{z} 580$ possibly can be found in C. orellanus or $C$. rubellus.

Department of Chemistry, Norwegian University of Science and Technology, Trondheim, Norway, for the preparation of the figures.

\section{REFERENCES}

[1] S. Grzymala, "Massenvergiftungen durch den Orangefuchsigen Hautkopf," Zeitschriftfür Pilzkunde, Vol. 23, 1957, pp. 139-142.

[2] I. Brondz, E. Nevo, S. P. Wasser, K. Høiland, F. P. Størmer and A. C. Brondz, "Method Development for in Vivo Analyses of Toxin Orellanine from the Toad Mushroom Cortinarius orellanus," Separation Science Europe 2011, 10-11 October 2011, The Royal Institution of Great Britain, London, Abstract 9.

[3] I. Brondz, E. Nevo, S. Wasser and A. Brondz, "A Direct Gas Chromatography-Mass Spectrometry (GC-MS) Method for the Detection of Orellanine Present in Stomach Content. Part I," Journal of Biophysical Chemistry, Vol. 3, No. 1, 2012, pp. 29-34. http://dx.doi.org/10.4236/jbpc.2012.31003

[4] I. Brondz and A. Brondz, "A High Performance Liquid Chromatography-Mass Spectrometry (HPLC-MS) Qualitative Detection Method Developed for in Vivo Analyses of Toxin Orellanine from the Cortinarius orellanus Fr. —Part II," ISRN Chromatography, Vol. 2012, 2012, Article ID 293830.

[5] I. Brondz, "Fungi, Classification of the Basidiomycetes," In: C. Batt and M. L. Tortorello, Eds., Encyclopedia of Food Microbiology, 2nd Edition, Elsevier, Oxford, 2014, in Press.

[6] T. Schumacher and K. Høiland, "Review Mushroom Poisoning Caused by Species of the Genus Cortinarius Fries," Archives of Toxicology, Vol. 53, 1983, pp. 87-106.

[7] I. R. Tebbett and B. Caddy, "Mushroom Toxins of Genus Cortinarius," Experientia, Vol. 40, No. 5, 1984, pp. 441446. http://dx.doi.org/10.1007/BF01952379

[8] L. Matthies and H. Laatsch, "Cortinarins in Cortinarius speciosissimus? A Critical Revision,” Experientia, Vol. 47, No. 6, 1991, pp. 634-640. http://dx.doi.org/10.1007/BF01949895

[9] M. Rohrmoser, M. Kirchmair, E. Feifel, A. Valli, R. Corradini, E. Pohanka, A. Rosenkranz and R. Pöder, "Orellanine Poisoning: Rapid Detection of the Fun-Gal Toxin in Renal Biopsy Material," Journal of Toxicology-Clinical Toxicology, Vol. 35, No. 1, 1997, pp. 63-66. http://dx.doi.org/10.3109/15563659709001167

[10] S. Rapior, N. Delpech, C. Andary and G. Huchhard, "Intoxication by Cortinarius orellanus: Detection and Assay of Orellanine in Biological Fluids and Renal Biopsies," Mycopathologia, Vol. 108, No. 3, 1989, pp. 155-161. http://dx.doi.org/10.1007/BF00436220

[11] W. Z. Antkowiak, R. Antkowiak, E. Wyrzykiewicz and G. Czerwiński, "Mass Spectral Fragmentation of Orellanine and Its Tetramethyl Ether with Regard to Their Facile Thermal and Photochemical Deoxygenation," Heterocycles, Vol. 39, No. 2, 1994, pp. 477-484. http://dx.doi.org/10.3987/COM-94-S(B)72

[12] J. Holmdahl, J. Ahlmen, S. Bergek, S. Lundberg, S.-A. Persson, "Isolation and Nephrotoxic Studies of Orellanine from the Mushroom Cortinarius speciosissmus," Toxicon, Vol. 25, No. 2, 1987, pp. 195-199. http://dx.doi.org/10.1016/0041-0101(87)90241-8

[13] P. Spiteller, M. Spiteller and W. Steglich, "Occurrence of the Fungal Toxin Orellanine as a Diglucoside and Investigation of Its Biosynthesis," Angewandte Chemie International Edition, Vol. 42, No. 25, 2003, pp. 2864-2867. http://dx.doi.org/10.1002/anie.200351066

[14] A. Herrmann, H. Hedman, J. Rosén, D. Jansson, B. Haraldsson and K.-E. Hellenæs, "Analysis of the Mashroom Nephrotoxin Orellanine and Its Glucosides," Journal of Natural Products, Vol. 75, No. 10, 2012, pp. 1690-1696. http://dx.doi.org/10.1021/np300135k

[15] I. Brondz, A. B. Fialkov and A. Amirav, "Analysis of Quinocide in Unprocessed Primaquine Diphosphate and Primaquine Diphosphate Tablets Using Gas Chromatog- 
raphy-Mass Spectrometry with Supersonic Molecular Beams," Journal of Chromatography A, Vol. 1216, No. 5, 2009, pp. 824-829. http://dx.doi.org/10.1016/j.chroma.2008.11.043

[16] A. Amirav, "What Can Be Improved in GC-MS-When Multi Benefits Are Transformed into a GC-MS Revolution," International Journal of Analytical Mass Spectrometry and Chromatography (IJAMSC), Vol. 1, No 1, 2013, pp. 31-47. http://dx.doi.org/10.4236/ijamsc.2013.11005

[17] H. Prast, E. R. Wemer, W. Pfaller and M. Moser, "Toxic Properties of the Mushroom Cortinarius orellanus," Archives of Toxicology, Vol. 62, No. 1, 1988, pp. 81-88. http://dx.doi.org/10.1007/BF00316263

[18] I. Brondz, D. Mantzeilas, U. Klein, M. N. Lebedeva, F. S. Mikhailitsyn, G. D. Souleimanov and D. Ekeberg, "The Main Contaminant of the Anti-Malarial Drug Primaquine is Its Positional Isomer," $3^{\text {rd }}$ International Symposium on Separation in BioSciences SBS 2003 A 100 Years of Chromatography, 13-18 May 2003, Moscow, Abstract p. $57,165$.

[19] I. Brondz, D. Mantzilas, U. Klein, D. Ekeberg, E. Hvattum, M. N. Lebedeva, F. S. Mikhailitsyn, G. D. Souleimanov and J. Røe, "Nature of the Main Contaminant in the Antimalaria Drug Primaquine Di-Phosphate: A Qualitative
Isomer Analysis," Chromatography B: Biomedical Sciences and Applications, Vol. 800, No. 1-2, 2004, pp. 211223.

[20] G. G. Coté and R. C. Crain, "Why Do Plants Have Phosphoinositides?" BioEssays, Vol. 16, No. 1, 1994, pp. 3946. http://dx.doi.org/10.1002/bies.950160106

[21] I. Brondz and A. Brondz, "Supercritical Fluid Chromatography-Mass Spectrometry (SFC-MS) and MADI-TOFMS of Heterocyclic Compounds with Trivalent and Pentavalent Nitrogen in Cough Relief Medical Forms Tuxi and Cosylan," American Journal of Analytical Chemistry, Vol. 3, No. 12A, 2012, pp. 870-876. http://dx.doi.org/10.4236/ajac.2012.312A115

[22] I. Brondz, "Mass Spectrometric Structure Elucidation of the Trivalent and Pentavalent Nitrogen Contaminants of Pholcodine in the Cough Relief Medical Form Tuxidrin," International Journal of Analytical Mass Spectrometry and Chromatography, Vol. 1, No. 1, 2013, pp. 5-10. http://dx.doi.org/10.4236/ijamsc.2013.11002

[23] G. M. Jones and J. A. Vale, "Mechanisms of Toxicity, Clinical Features, and Management of Diquat Poisoning: A Review," Journal of Toxicology-Clinical Toxicology, Vol. 38, No. 2, 2000, pp. 123-128. http://dx.doi.org/10.1081/CLT-100100926 